\title{
Migration and Dietary Diversity Changes among the Students: Case Study of the University of Delhi in India
}

Saumya Mishra ${ }^{\dagger}$ and Subhash Anand ${ }^{* *}$

\section{Abstract}

A large number of students migrate every year to the University of Delhi to pursue higher studies. Most of these students find accommodation within the vicinity of the university, that is North Campus of the university. Change in their daily diets, induced by the migration, therefore, becomes a critical aspect of determining their physical and mental well-being. The paper aims to examine the changes in their dietary diversity after migration. The principal focus is to analyse the comparative qualitative differences in the diet of the students before and after migration to the University of Delhi. The focus group for the research work comprises randomly selected migrant students from different parts of India, presently living in the North Campus of Delhi. For the primary survey, 100 respondents have been selected from four localities within the North Campus (Vijay Nagar, Malka Ganj, Kamla Nagar and Guru Tegh Bahadur Nagar) to get first-hand information and opinions. Both descriptive and inferential statistical techniques have been applied to identify the relationship between socio-economic and demographic features of the respondents, and the changes in their dietary diversity have been examined. The findings demonstrate an alarming trend being prevalent across all the four localities-in the consumption of nuts, dry \& fresh fruits and vegetables along with the simultaneous trend of a significant increase in the fast-food consumption. The extent of the change varied significantly across the four localities. The highest decline in dietary diversity was observed in Vijay Nagar, whereas Kamla Nagar experienced the least changes in dietary diversity.

Keywords: Migration; Dietary; Consumption; Students; University of Delhi, Delhi; India

\footnotetext{
' Saumya Mishra, Former Student, Department of Geography, Delhi School of Economics, University of Delhi, Delhi110007, India. Email: msaumya55@gmail.com

${ }^{*}$ Corresponding Author, Email: sanandpvs@gmail.com

*Associate Professor, Department of Geography, Delhi School of Economics, University of Delhi, Delhi- 110007, India (C) 2020 Mishra \& Anand. This is an Open Access article distributed under the terms of the Creative Commons Attribution License (http://creativecommons.org/licenses/by/2.0), which permits unrestricted use, distribution, and reproduction in any medium, provided the original work is properly cited.
} 


\section{Introduction}

Migration, as a process, is aimed at the betterment of the quality of life and livelihood, and access to hitherto unavailable opportunities in the place of origin. Lee (1966) identified that there are many push and pull factors responsible for migration, education being one of them. One of the Ravenstein's migration laws postulates that the leading causes of migration are economical. Recently, Portuguese universities have reported a massive increase in the number of migrant students in their student bodies (Nada and Araújo, 2017). Education, as an indirect determinant of economic well-being, can be seen within the framework of economic migration encouraging student migration (Grigg, 1977). Non-availability of required infrastructure, coupled with the lack of access to quality education in the area of origin is a major driving force of student migration (Pavithra, 2019). India has experienced a gradual increase in the number of students migrating to different places in recent years. The flow of students has proliferated in the last few decades (Brezis and Soueri, 2011). According to the Census of India (2011), about 3.29 million men and 2.1.6 million women have migrated internally in India in search of better education opportunities (Pavithra, 2019). The increasing proportion of diverse population groups, in terms of the area of origin, gender, age, class, culture, and individual preferences, initiates a process, encouraging the mutual exchange of ideas and values among different migrant groups and the local population, dietary components being one of them.

Dietary pattern analysis is an emerging area of research (Green et al., 2016). The last two centuries have experienced a dramatic change in diets all across the world, both in developed and developing countries. The transition from living in the comfort of home to independent living at a relatively newer place brings both opportunity and challenges for students. It is especially true for a country like India, which exhibits substantial areal variations and traditional vegetarian patterns (Green et al.,
2016) in eating habits and dietary components. The daily diet of students faces various challenges in this case. This trend remains relevant for many sections, including the migrants. In terms of food habits, immigrants are influenced by the food culture of the majority, leading to changes in their dietary habits (Abouta et al., 2002). It is usually difficult for the migrants to incorporate the food habits and change their diet according to the newer environment in the host area.

It is critical to developing good eating habits during this period because such behaviours usually continue throughout life (Shin, 2018). Therefore, leaving the comfort of home, with a range of eating options and moving to a new place, with different eating options combined with the unfamiliarity to many of these options, leads to changes in dietary diversity of the migrants. Sustainable Development Goal- 2 aims at ending the hunger and malnutrition, ensuring access to all for healthy and nutritious food (Kumar et al., 2018) is very significant in this respect. Dietary diversity, therefore, holds a high value for students because not only their physical and cognitive development but also the academic performance is dependent on daily nutrition.

Consequently, a diverse diet, comprised of different food groups, is indispensable to meet the daily nutrition requirements of the students. The Indian government has taken several efforts in terms of policies and programmes for the nutritional in females, children vulnerable groups of the society (Shankar, 2017). In India, University of Delhi is one of the best institutions for higher studies, attracts a large number of students from different parts of the country. Their regional, socio-economic and cultural diversity resulting from migration, therefore provides an ideal landscape to explore the resultant changes in the diet of students. The present paper attempts to critically analyse the migration led changes in the dietary diversity of the students at the University of Delhi. The next section reviews relevant literature linked to the study to address the knowledge gap. 


\section{Review of Literature}

As migration introduces a person to a new environment, both socially and culturally, they often experience a loss of their traditional food options and culinary practices. The migration of individuals, thereby, is also the migration from traditional food options and culinary practices (Kershen, 2002). Ruel (2003) defines dietary diversity as the number of different foods or food groups consumed over a given reference period. Oussedik (2012) concluded that single and comparatively younger and new migrants take less healthy diets, skewed towards convenience foods. On the other hand, the older migrants comparatively take healthier diets, and a big part of their diet is based on the foods and practices of their area of origin. Changes in diet are also closely related to economic status, as found by Akerele and Odeniyi (2015) who established a positive correlation between the household income, and the level of dietary diversity, and a negative correlation between food prices and the daily dietary diversity. Batta et al., (2016) analysed that students getting primary education suffer more from vitamin deficiencies, whereas this trend is not very dominant among college-going students. Shankar et al., (2017) correlated malnutrition with the family dynamics, and policies \& programs focused on preventing malnutrition. Khadka (2018) observed that instead of migration as a dominant factor, diet transformation in urban areas results from the increase in commercial food value chains, changing food preferences and choices because of globalisation and information and communication technologies. Nada and Araújo (2018) found that the different situation in which students deal with the issues and challenges of living and studying in a foreign country is highly diverse in nature.

Similarly, Abraham (2018) analysed that there is little to no impact on the knowledge about the unhealthiness of fast food and junk food on the frequency of fast food consumption by college students. Shin (2018) observed that it is not only the transition to a new academic level that challenges the food habits of college students but also, and, more importantly, the freedom and independence to choose from various available food choices which affect the diet composition of the students. In a similar context, Rukmini (2019) found that on an average, Indians consume more carbohydrates, while the consumption of protein, fruits and vegetables is much less than the dietary recommendations by Lancet Commission. This study, however, links migration and diet to examine the dietary changes among the migrant students pursuing studies at the University of Delhi, India.

A description of the study area and the methodological approach deployed for this study are discussed in the following sections.

\section{Study Area}

Delhi is located in the floodplains of Yamuna and straddles, both the banks of the Yamuna river (Anand, 2010). North campus of the University of Delhi is located in the district of North Delhi (Census of India, 2011). The campus extends from $28^{\circ} 35^{\prime} 3.66^{\prime \prime} \mathrm{N}$ latitude to $77^{\circ} 09^{\prime} 46.62^{\prime \prime} \mathrm{E}$ longitude (Figure1). Delhi University is one of the largest universities in India with 16 faculties, more than 80 academic departments (University of Delhi, 2019). The ten colleges in the North Campus are situated close to each other in an area that starts from Vishwavidyalaya Metro Station and ends at the part of Bungalow Road which joins entrance area of Hansraj College with the back gate of Kirori Mal College. The Northern Ridge, a hilly forested area, also famously known as 'Green Lungs' of North Delhi, surrounds the western and northwestern part of the city of Delhi, running from Mall Road along with the North Campus. With a total area of 61 square kilometres, that is 4.1 per cent of the total area of Delhi. 


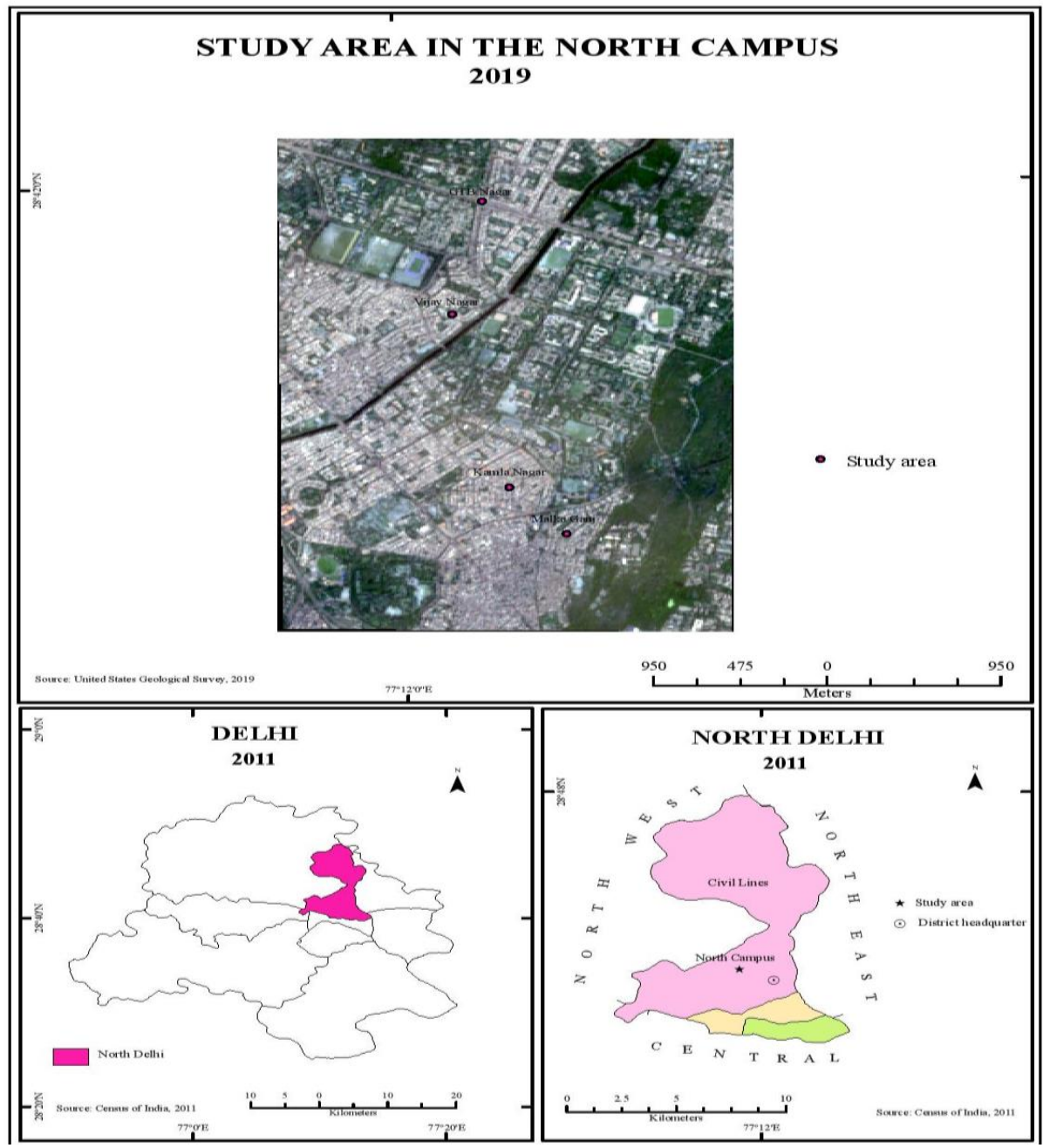

Figure 1: Location Map of Study Area

Source: Census of India, 2011

\section{Database and Methodology}

The study aims to examine the relationship between student migration and changes in their dietary diversity. The principal focus is to analyse the qualitative differences between the diet of the students before and after migration. Research, therefore, takes into account the comparative study of the area of origin and area of destination in the form of student responses through their comparative insights. A variety of secondary data was gathered from Census reports, international organisations reports, peer-reviewed studies, research papers, articles, and newspapers and websites.

Several pieces of research have indicated that focus groups are an appropriate research method for the studying eating habits, especially among the students (LaCaille et al., 2011; Deliens et al., 2014). The target population for the research was inter-state migrant college students in the age group of 18 to 25 years, presently enrolled in various courses of Delhi University and living in the north campus of Delhi University. Four localities (Vijay Nagar, Malka Ganj, Kamla Nagar and GTB Nagar) within the north campus, which house these migrant students, were selected as the area of study. Non-purposive sampling was engaged to select a sample size of 25 from each of the four localities, forming the total sample size as 100 respondents. The responses of the close-ended questions were coded and entered into the Statistical Package for the Social Sciences (SPSS) software. Both descriptive and inferential statistical techniques were used to analyse the quantitative data. Correlation and linear regression analysis were used to identify the relationships between socio-economic and demographic features of the respondents and 
the changes in their dietary components and related variables.

\section{Results and Discussion}

Dietary diversity can be used as a proxy measure of food security (Oussedik, 2012). A rich diet, which includes diverse food groups, is characterised usually with adequate nutrients. Such a diet ensures greater nutrition of consumers and by reflecting food security, provides an indicator of food security. Dietary diversity is also associated with the quality of diet, and nutrient as well as energy intake. Therefore, it can be said that dietary diversity is associated with malnutrition as well. This is mainly reflected in the patterns of increasing incidences of cardiovascular diseases, diabetes, obesity, and cancer etc. that are positively correlated with the changes in the dietary pattern (Taskar et al., 2007).

\section{Source of Food in the Destination Area}

Source of food is an essential determinant of changes in dietary diversity. It is because every food source has its own pattern of food supply, defined food components and a confined area of experiment with diversity in the supply pattern. The source of food also affects and in turn is affected by food consumption behaviour of the local population as well as the affordability of the food (Kearney, 2010). A more diverse and nutritionally balanced diet and higher levels of food hygiene are associated with better health (Kulkarni and Gaiha, 2010). The mess of the hostel or paying guest (PG) or the tiffin service is the chief source of food for college students in the area of north campus, followed by the street food. Street food provides the most affordable and readily available source of food while also being a convenience food as it provides readyto-eat food. Self-cooked food is the third most prevalent food source. Even though it ranks third, its prevalence is less than almost half of the food provided by messes and tiffin service. This is attributed to the reasons like time constraints with students as well as not knowing or not liking to cook the food on their own. The fourth most important source is college canteen food which enjoys the dominance close to that of self-cooked food. It is evident that college canteen, being located within the premises of the colleges, provides food at affordable prices to the students, which are quite popular among the students who spend majority of their time in college campuses. This is followed by the food cooked by monthly waged cooks or maids who are usually hired by the students living in flats or separate rooms instead of hostels. The next important source is the food served in restaurants. Packaged food comes next, which provides ready-to-eat food types and is popular among the students who have hectic schedules. Processed food and semi-processed food, surprisingly, are a comparatively less important source of food for the migrant students in north campus. This might be due to the awareness among students that such food is less nutritious and unhealthy due to high fat, sugar or salt content (Figure 2). 


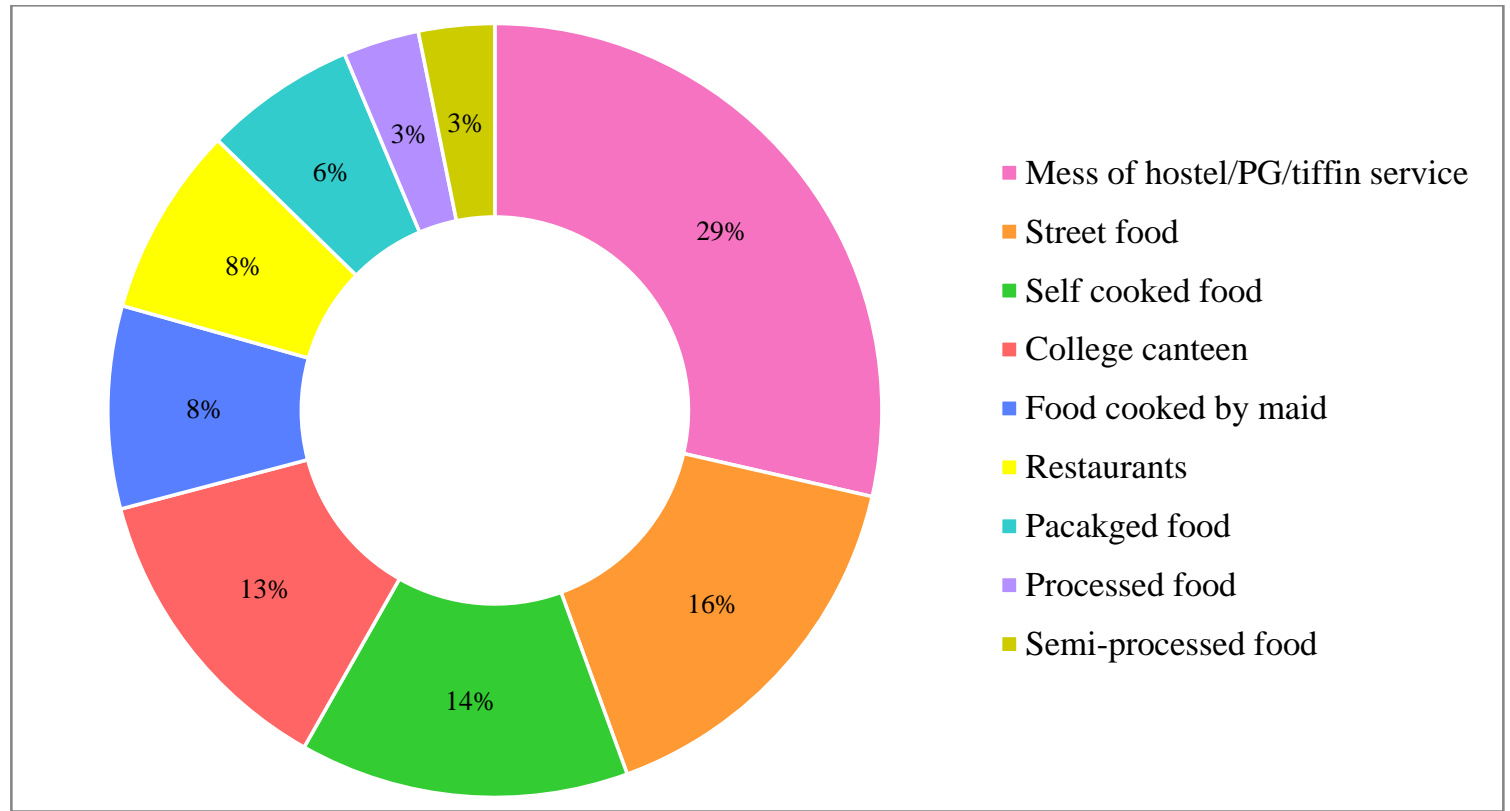

Figure 2: Major Sources of Food for the Students in the North Campus, Delhi University Source: Primary Survey, 2019

\section{Post Migration Changes in the Total Dietary Diversity of the Students}

The changes in dietary diversity post-migration can be analysed by measuring the Individual Dietary Diversity Scores (IDDS) before and after migration. Table 1 depicts the average IDDS of the students before and after migration in the Delhi University. The result indicates that the average dietary diversity before migration is higher than that of after migration. A reduction of 0.57 in the IDDS is observed after migration (Table 1).

\section{Table 1: Individual Dietary Diversity Scores of the students before and after the Migration}

\begin{tabular}{|l|c|}
\hline Phase & Average Individual Dietary Diversity Score (IDDS) \\
\hline Before Migration & 10.73 \\
\hline After Migration & 10.16 \\
\hline Change Experienced after Migration & 0.57
\end{tabular}

The inter-regional differences in the four localities suggest that dietary diversity has reduced after migration in all the four regions. With an average dietary diversity score of 10.13 and 10.16 before migration and after migration, respectively, Vijay Nagar shows the highest reduction in dietary diversity post-migration. Malka Ganj, near to Kirori Mal and Hansraj college with a difference of 0.12 in average dietary diversity before and after migration, represents the least change amongst the four regions. Rest two regions, namely Kamla Nagar and GTB Nagar depict a moderate reduction in dietary diversity, with the average difference of 0.84 and 0.2 in IDDS in pre and post-migration phases.
To analyse a more outstanding picture of changes in dietary diversity, the need to emphasise upon specific food groups cannot be denied. The general trend shows a decline in the weekly intake of the majority of the food groups among the migrant students' migration. The highest amount of reduction is seen in the case of nuts and dry fruits, with a total of 29 per cent reduction in weekly food intake, followed by white roots and tubers and fresh fruits. Twentyone per cent students do not consume white roots and tubers weekly after migration, while 17 per cent of the students consume fresh fruits less frequently after the migration. Milk \& milkbased products and sweets showed a moderate reduction of 11 per cent each in the weekly 
intake. Lowest reduction of 1 per cent is observed in the case of cereals, followed by vegetables, oils and fats, fishes and seafood, and pulses.

On the contrary, students reported the highest increase in the intake of fast food after migration. Thirty-six per cent of the students consume fast food more frequently after migration. Contrary to the general trend, four food groups, namely meat, eggs, spices and beverages, and fast food depict an increase in the intake after migration. After migration, the consumption of eggs has increased by 15 per cent, followed by spices and beverages, and meat with an increase of 4 per cent and 2 per cent respectively (Figure 3 ).

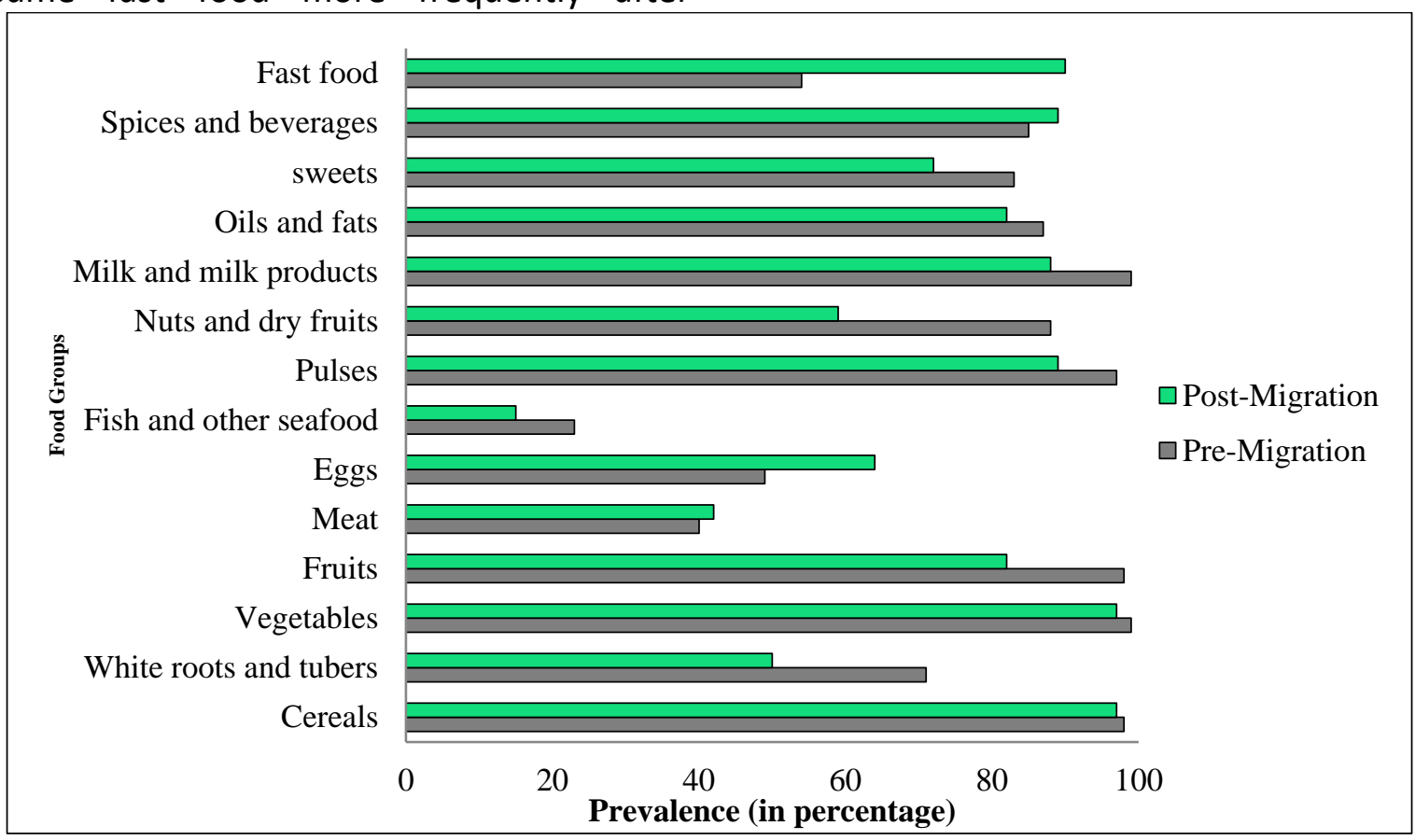

Figure 3: Prevalence of Weekly Food Intake of Food Groups before and after Migration Source: Primary Survey, 2019

\section{Frequency of Fresh Fruit and Vegetable Consumption after Migration}

Fruits and vegetables, with low fat, sugar and sodium contents, are a necessary part of a healthy and balanced diet. Hence, it is vital to understand the frequency of fresh fruit and vegetable consumption among the students' post-migration. The daily intake of fresh fruit and vegetable has an equal proportion of the students in both Malka Ganj and GTB Nagar that is 28 per cent in each. It is lowest in Kamla Nagar where only 8 per cent students consume fresh fruits and vegetables on a daily basis. Alternative consumption of fresh fruits and vegetables in a week is highest for the students in Vijay Nagar with 36 per cent proportion, followed by the Kamla Nagar. The lowest alternative consumption is observed in GTB Nagar, with only 16 per cent of students consuming fruits and vegetables alternatively. Sixteen per cent student population in the three areas except Malka Ganj reported fruit and vegetable consumption twice in a week. Once in a week consumption is equally observed in Kamla Nagar and GTB Nagar with 16 per cent of consuming population while the proportion was only 4 per cent in Vijay Nagar. A percentage as high as 24 per cent in GTB Nagar and Kamla Nagar reported rare consumption of fruits and vegetables in a week, whereas 20 per cent students in Vijay Nagar and Malka Ganj responded to have the same tendency. Once in a week consumption is the smallest category with only 12 per cent of the students falling in it (Figure 4). 


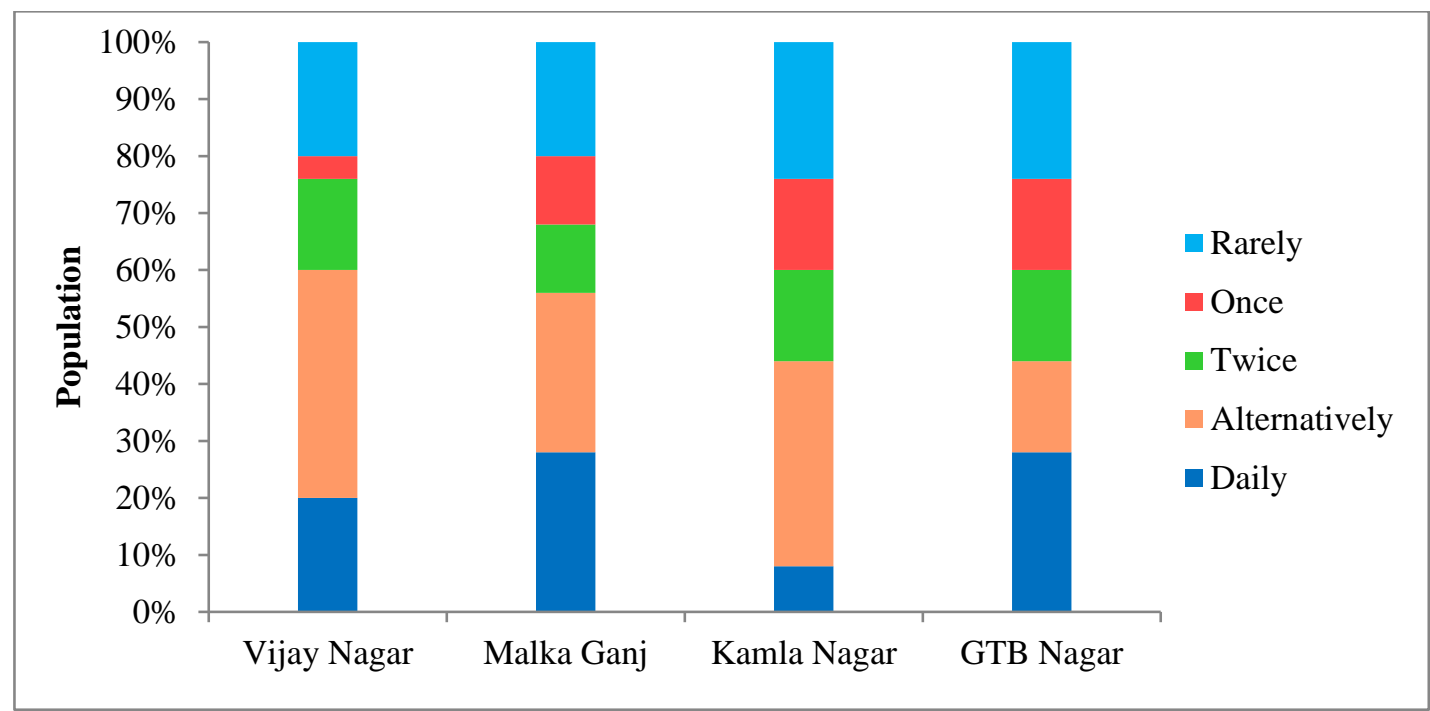

Figure 4: Weekly Frequency of Fresh Fruit and Vegetable Consumption after Migration Source: Primary Survey, 2019

\section{Frequency of Fast Food Consumption after Migration}

It has been observed that the consumption of fast food has increased tremendously among college students in North Campus. The frequent consumption of fast food is associated with health risks such as obesity, hypertension, and cardiovascular diseases etc. (Mahdizadeh et al., 2016). The leading factor responsible for the college students is the readily available nature of fast foods in the Campus and the vicinity areas. Additionally, the quick-too-prepare nature of fast foods makes them widely preferred by students as they have a crunch of time due to hectic academic schedules. Third next important, and also relatively the most recently emerged factor, is the online delivery apps like Zomato, Foodpanda and others which bring the food at the door of the students, providing them with the convenience of not going to market to get food. Attractive advertisements for fast food which tempt students to buy fast food is also a significant factor. These advertisements are usually accompanied by various offers by fastfood chains to lure students and other individuals and families.
It has been found that after migration, almost two-thirds of the students reported the increase in fast food consumption. Eighteen per cent population recorded no changes in the fast-food consumption after migration, whereas the rest 8 per cent population remained unclear. In the four localities of the study area, daily consumption of fast-food is highest among the students in Vijay Nagar where six students consume fast food daily, followed by GTB Nagar with five students consuming fast food daily. It is lowest in Malka Ganj with only two students who consume fast food on a daily basis. Alternative fast food consumption in a week is highest in Vijay Nagar, followed by GTB Nagar. Twice in a week consumption is highest in GTB Nagar with a reporting student population of eight, followed by Vijay Nagar and Kamla Nagar with four such students in each. Rare fast food consumption, which is once or twice in a month, is most prevalent in Malka Ganj, followed by Kamla Nagar. It is least prevalent in GTB Nagar, where only four students reported consuming fast food rarely (Figure 5). 


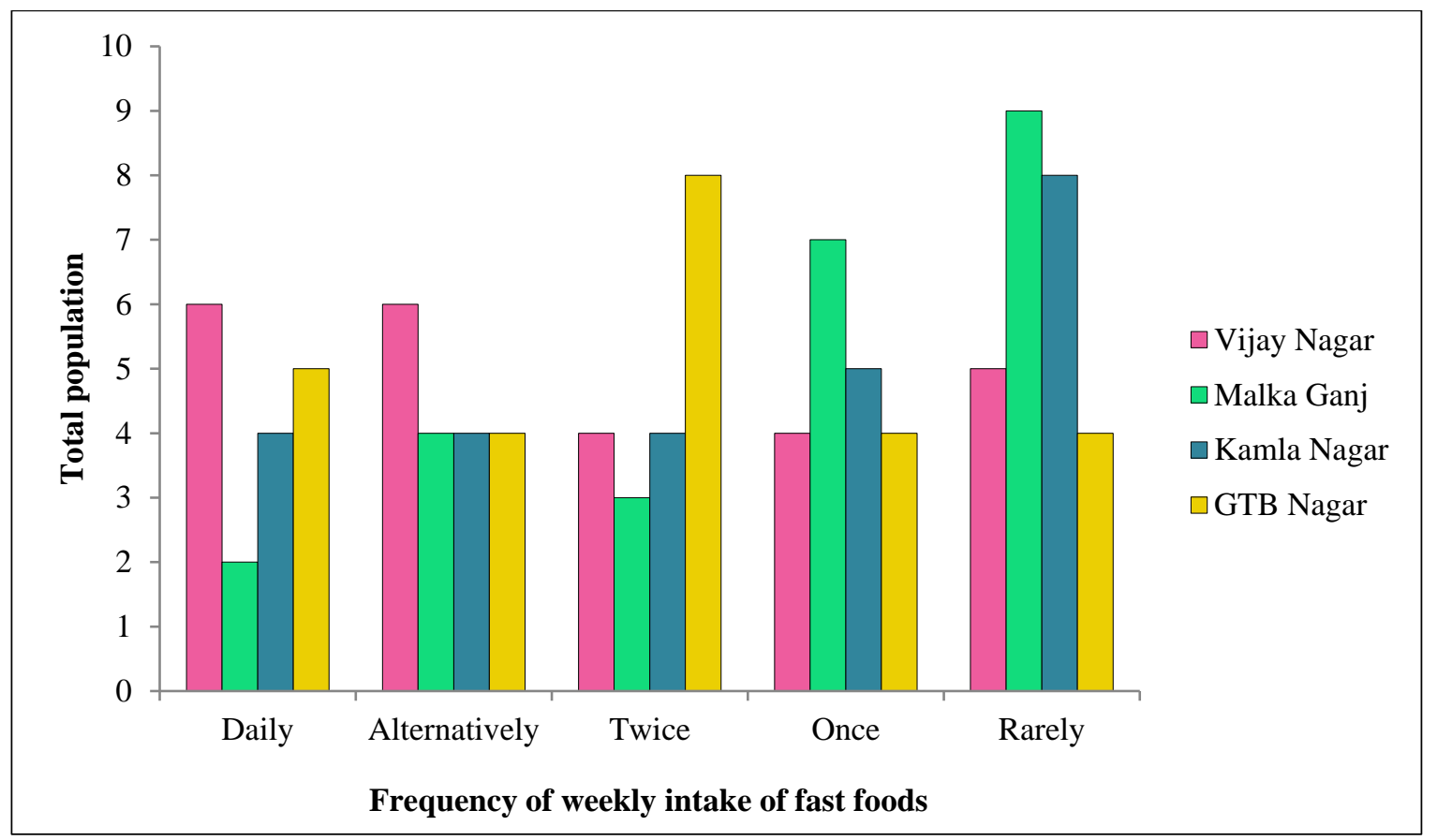

Figure 5: Weekly Intake of Fast Foods by the Students Source: Primary Survey, 2019

\section{Differentials based on the Duration of Migration}

Several studies explain the adverse impacts of long term migration on the food consumption behaviour of the migrants. Thus, it is significant to explore the impacts of short term and long term migration on the dietary diversity of the students. Table 2 stratifies the college students in the study area based on the duration of their migration in Delhi.

\section{Table 2: Duration of Migration of the Students in the Study Area}

\begin{tabular}{|c|c|c|c|c|c|}
\hline Area & $\begin{array}{l}\text { Less than } 1 \\
\text { Year }\end{array}$ & 1-3 Years & 3-5 Years & $\begin{array}{l}\text { More than } 5 \\
\text { years }\end{array}$ & Total \\
\hline Vijay Nagar & 7 & 11 & 2 & 5 & 25 \\
\hline Malka Ganj & 1 & 8 & 13 & 3 & 25 \\
\hline Kamla Nagar & 2 & 10 & 10 & 3 & 25 \\
\hline GTB Nagar & 1 & 5 & 13 & 6 & 25 \\
\hline Total & 11 & 34 & 38 & 17 & 100 \\
\hline
\end{tabular}

There exists a clearly visible trend of decrease in dietary diversity with the increase in the length of migration. With the correlation of -0.987 and Coefficient of determination as 0.974 , there is strong negative correlation between the duration of migration and the decrease in dietary diversity. The steepest fall in dietary diversity is experienced by the students who have migrated within short-span of one year, followed by those who are migrants for one to three years. The change in IDDS is about 45 per cent less for the students who are staying in North Campus from three to five years than those with one to three years of migration duration. The change is lowest for the students with five years or more of migration duration. These students encounter 67 per cent lesser changes than the students who are staying in North Campus from less than one year (Figure $6)$. 


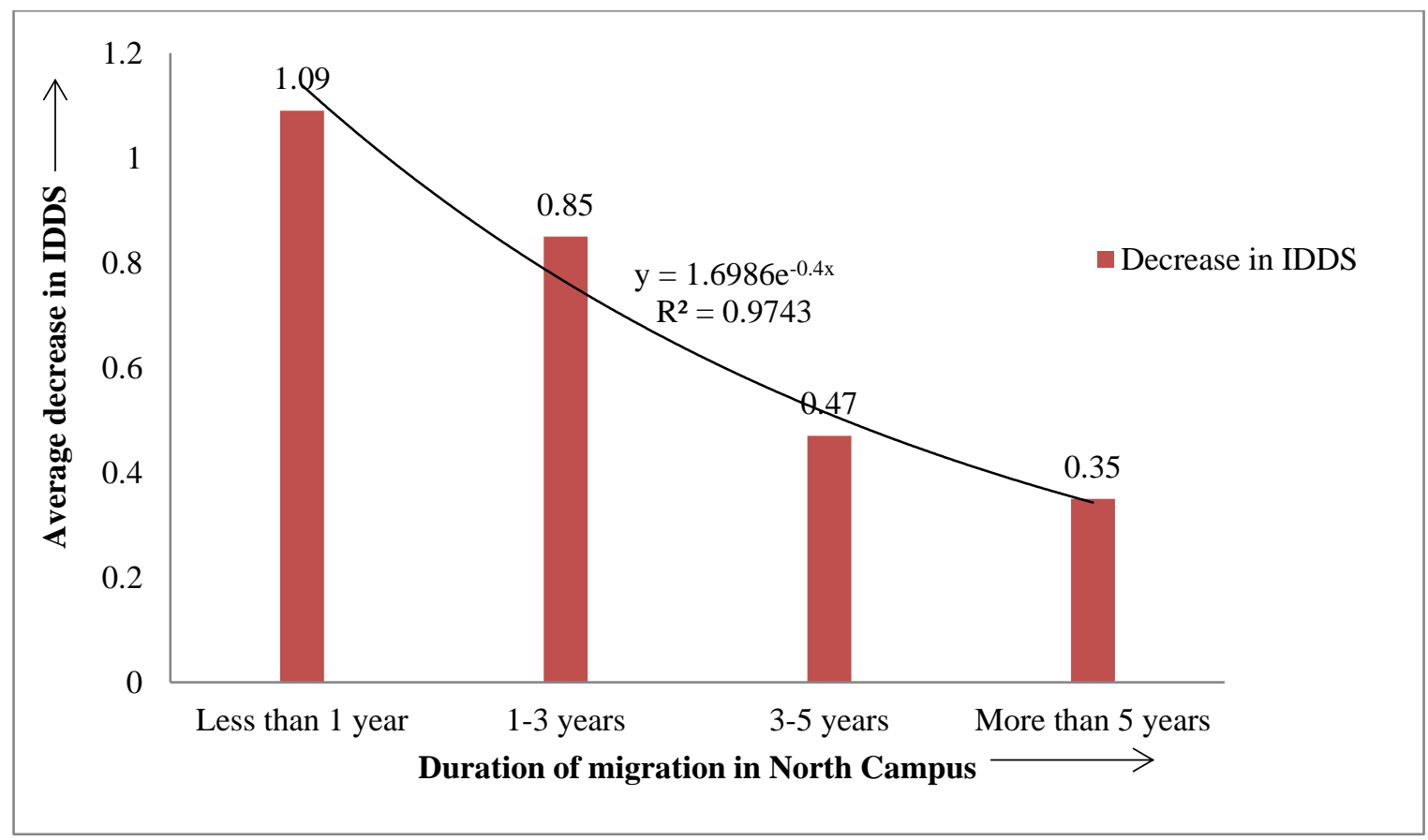

Figure 6: Relationship between the Duration of Migration and the Change in Dietary Diversity Source: Primary Survey, 2019

\section{Non-Consumption of the Local Food in the Place of Destination}

Food is not only a means of survival but also a means of cultural expression and a marker of cultural identity. As migration brings a new environment, both social and cultural, to the migrants, they often experience a loss of their traditional food options and culinary practices. The migration of individuals, thereby, is also the migration from traditional food options and culinary practices (Kershen, 2002). Almost 90 per cent of the students reported the inability to consume one or more local food of their hometown in Delhi. Many factors play a role in causing it. Out of these, the most crucial factor is the non-availability of the local food. Immigration hubs, like Delhi, usually offer a hybrid cultural experience instead of the original. 21 per cent students reported the cultural appropriation and massive changes in the forms of traditional food. A subsequent, crucial factor is non-accessibility and nonaffordability of the traditional local food. The stigmatisation of the local food in driving students away from consuming their traditional food in Delhi was the least prevalent driving factor (Table 3).

\section{Table 3: Reasons for Non-Consumption of the Local Food by the Migrant Students}

\begin{tabular}{|l|l|}
\hline Reasons & Affected Student Population (in percentage) \\
\hline Non-availability & 32 \\
\hline Non-affordability & 11 \\
\hline Non-accessibility & 18 \\
\hline $\begin{array}{l}\text { Cultural appropriation and massive changes } \\
\text { in the form of traditional food }\end{array}$ & 21 \\
\hline The stigmatisation of the traditional food & 2 \\
\hline Other reasons & 16 \\
\hline Source: Primary Survey, 2019 & \\
\hline
\end{tabular}




\section{Conclusion}

The study on the post-migration changes in dietary diversity of the college students reveals interesting patterns and relationships. The findings point to an alarming trend that remains prevalent throughout the study and across all the four localities was the reduction in the consumption of nuts, dry \& fresh fruits and vegetables along with the simultaneous increase in the fast-food consumption. The highest decline in dietary diversity was observed in Vijay Nagar, whereas Kamla Nagar experienced the least changes in dietary diversity. It is very likely that the shifts in dietary diversity are also emanating from the availability and accessibility to new food options, which were hitherto unavailable to students owing to their culture, familial surveillance, traditions and taboos linked with the meat and egg consumption.

Due to crunch of time among college students, fast foods, being relatively cheaper and quickto-prepare, act as convenience food. This is giving rise to unhealthy shifts in dietary diversity of the students. The non-availability of the local or traditional food of the migrant students in Delhi, such as- curry of Pothia fish (Bihar), leafy vegetables like Onger, Owin, Obu Olu (North Eastern states), was the leading factor which reduced the diversity in their diets by removing the food options that were present in the place of origin. There is a trend of increase in dietary diversity with increasing levels of educational qualification. Duration of migration, on the other hand, has a negative correlation with the change in dietary diversity. It is possibly due to a greater degree of adaptation in Delhi by the students with longer duration of migration.

The overall non-uniformity in the degree of influence enjoyed by the socio-economic and demographic determinants in causing a change in dietary diversity emphasises the need of the more inclusive strategies to incorporate these variations in order to ensure healthier food options being available for the students. Nutrition education should also be mainstreamed into university curriculums in order to ensure good health and wellbeing of the students for the sustainable future.

\section{References}

Abouta, J.S., Patterson, R.E., Neuhouser, M.L. and Elder J. (2002). Dietary acculturation: applications to nutrition research and dietetics. Journal of the American Dietetic Association, 102, 1105-18. https://doi.org/10.1016/S0002 8223 (02)90247-6

Abraham, S., Shin, J. Y., \& Norigea, B. R. (2018). College students eating habits and knowledge of nutritional requirements. Journal of Nutrition and Human Health, 2(1), 13-17.

https://doi.org/10.35841/nutrition-humanhealth.2.1.13-17.

Akerele, D. \& Odeniyi, K. A. (2015). Demand for diverse diets: Evidence from Nigeria.

89th Annual Conference of the Agricultural Economic Society, University of Warwick, UK

Anand, S. (2010). Solid Waste Management. Mittal Publications.

Batta, M. Gupta, N., Goyal, G., \& Jain, A. (2016). Vitamin deficiency prevalence in primary school children in Punjab. India, International Journal of Research in Medical Sciences, 4(12), 5176-

5179. http://dx.doi.org/10.18203/23206012.ijrms20164011

Brezis, E. \& Soueri, A. (2011). Why do Students Migrate? Where do they Migrate to?. Working Papers 25, AlmaL aurealnter-University Consortium. https://ideas.repec.org/p/laa/wpaper/25.html

Census. (2011). North Delhi District: Census 2011-2019 data.

https://www.census2011.co.in/census/district/ 169-north-delhi.html

Deliens, T., Clarys, P., De Bourdeaudhuij, I. et al. (2014). Determinants of eating behaviour in university students: a qualitative study using focus group discussions. BMC Public Health, 14, 53 https://doi.org/10.1186/1471-2458-14-53

District Census Handbook. (2011). District census handbook of N.C.T. of Delhi, Census of India. New Delhi.

Green, R., Milner, J., Joy, E. J.M. et al.,(2016).

Dietary patterns in India: A systematic review. 
The British Journal of Nutrition, 116 (1). 142148.

https://www.ncbi.nlm.nih.gov/pmc/articles/P MC4890343/

Grigg, D. B. (1977). E. G. Ravenstein and the "laws of migration". Journal of Historical Geography, 3(1), 41-54.

https://www.sciencedirect.com/science/article /abs/pii/0305748877901438

Kearney, J. (2010). Food consumption trends and drivers. Philosophical transactions of the Royal Society of London. Series B, Biological Sciences, 365(1554), 2793-2807. https://pubmed.ncbi.nlm.nih.gov/20713385/

Kershen, A. J. (2002). Food in the Migrant Experience, Routledge.

Khadka, S. (2018). Migration and food security: Challenges and opportunities for India. Down To Earth. https://www.downtoearth.org.in/food Kulkarni, V.S. \& Gaiha, R. (2010). Dietary transition in India, Center for the Advanced Study of India, University of Pennsylvania. https://casi.sas.upenn.edu/iit/kulkarnigaiha

Kumar, N., Anand, S. and Singh, J. (2018). Understating the status of food Security: A Case Study of Haryana, The Horizon - A Journal of Social Sciences, IX (1), 1-16 https://thehorizonjournal.in/wpcontent/uploads/2019/04/January-2018.pdf

Lacaille, L.J., Nichols, K., Krambeer, J. and Pedersen, J., (2011). Psychosocial and environmental determinants of eating behaviors, physical activity, and weight change among college students: A qualitative analysis. Journal of American College Health, 59, 531-538. https://doi.org/10.1080/07448481.2010.52385 5

Lee, E. S. (1966). A Theory of Migration, Demography, 3(1),47-57.

https://www.jstor.org/stable/2060063

Mahdizadeh, H. A, Manhaz S., Montazeri A., Shojaeizadeh D, Nejat S, Farahani F.K., \& Djazayeri, A. (2016). Factors Influencing FastFood Consumption Among Adolescents in
Tehran: A Qualitative Study. Iranian Red Crescent Medical Journal, 18(3). https://www.ncbi.nlm.nih.gov/pmc/articles/P MC4884438/

Nada, C. I. \& Araújo, H. C. (2017). The multicultural experience of international students in Portugal: A narrative approach. Journal for Multicultural Education, 11 (3), 17688. https://doi.org/10.1108/JME-09-2016-0049. Nada, C. I. \& Araújo, H. C. (2018). Migration and education: A narrative approach to the experience of foreign students in Portugal. London Review of Education, 16 (2), 308-324. https://doi.org/10.18546/LRE.16.2.10.

Oussedik, S. (2012). Food and Cuisine: Part of the Migration Process. Institut Europeu de la Mediterrània.

Pavithra, K. M. (2019). 1 out of every 100 migrants in India move for education, https://facty.in/1-out-of-every-100-migrantsin-india-move-for-education/

Ruel, M. T. (2003). Operationalizing dietary diversity: A review of measurement issues and research priorities. The Journal of Nutrition, 133 (11). 3911S-3926S. Oxford University Press. https://academic.oup.com/jn/article/133/11/3 911S/4818042\#111984609

Rukmini, S. (2019). Indians are a long way away from an ideal diet. Livemint.

https://www.livemint.com/science/health/indi ans-are-a-long-way-away-from-an-ideal-diet1548618112928.html

Shankar, B. (2017). Dietary and nutritional change in India: Implications for strategies, policies and interventions, Wiley Annals of the New York Academy of Sciences. http://dx.doi.org/10.1111/nyas.13324

Sogari, G., Velez-Argumedo, C., Gomez, M.I. and Mora, C. (2018). College students and eating habits: A study using an ecological model for healthy behavior, Nutrients, 10(12), 1823. https://doi.org/10.3390/nu10121823

Taskar, Priya D., Nicklas, T.A. \& Berenson, G. (2007). Does food group consumption vary by differences in socioeconomic, demographic, and lifestyle factors in young adults? The 
Bogalusa Heart Study. Journal of American Diet Association, 107 (2), 223-234.

https://doi.org/10.1016/j.jada.2006.11.004

University of Delhi. (2019). About University of Delhi.

http://www.du.ac.in/du/index.php?page=abou t-du-2

\section{Conflict of Interest Statement}

There is no potential conflict of interest for the manuscript.

\section{Acknowledgement Statement}

Both authors are very thankful to the Department of Geography, University of Delhi, Ratan Tata Library, for providing the necessary infrastructures for writing the manuscript.
Authors are highly obliged and thankful to the anonymous reviewers for their positive critical comments which helped in improving the manuscript.

\section{Author Contribution Statement}

Saumya Mishra, former student of Department of Geography, Delhi School of Economics, University of Delhi, India: Conceptualisation, methodology, formal analysis, investigation, writing \& editing, checking for references.

\section{Subhash Anand, Associate Professor in} Geography, Delhi School of Economics, University of Delhi, India (Corresponding author): Supervision, validation, reviewing and editing of the final draft. 